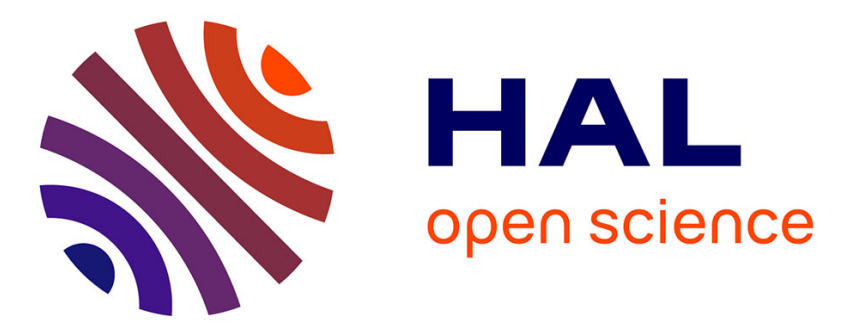

\title{
Destabilization of the Nematic Phase of Clay Nanosheet Suspensions by Polymer Adsorption
}

Karin El Rifaii, Henricus H Wensink, Thomas Bizien, Jean-Christophe P Gabriel, Laurent Michot, Patrick Davidson

\section{- To cite this version:}

Karin El Rifaii, Henricus H Wensink, Thomas Bizien, Jean-Christophe P Gabriel, Laurent Michot, et al.. Destabilization of the Nematic Phase of Clay Nanosheet Suspensions by Polymer Adsorption. Langmuir, 2020, 36 (42), pp.12563-12571. 10.1021/acs.langmuir.0c02084 • hal-03325014

\section{HAL Id: hal-03325014 \\ https://hal.science/hal-03325014}

Submitted on 24 Aug 2021

HAL is a multi-disciplinary open access archive for the deposit and dissemination of scientific research documents, whether they are published or not. The documents may come from teaching and research institutions in France or abroad, or from public or private research centers.
L'archive ouverte pluridisciplinaire HAL, est destinée au dépôt et à la diffusion de documents scientifiques de niveau recherche, publiés ou non, émanant des établissements d'enseignement et de recherche français ou étrangers, des laboratoires publics ou privés. 


\title{
Destabilization of the nematic phase of clay
}

\section{nanosheet suspensions by polymer adsorption}

\author{
Karin El Rifaii, ${ }^{\S}$ Henricus H. Wensink, ${ }^{\S}$ Thomas Bizien, ${ }^{\#}$ Jean-Christophe P. Gabriel, ${ }^{\dagger}$ Laurent \\ Michot, ${ }^{* \perp}$ Patrick Davidson $* \mathcal{S}$ \\ ${ }^{\S}$ Université Paris-Saclay, CNRS, Laboratoire de Physique des Solides, 91405, Orsay, France. \\ ${ }^{\#}$ SWING beamline, SOLEIL Synchrotron, 91192 Gif-sur-Yvette, France \\ ${ }^{\dagger}$ Université Paris-Saclay, CEA, CNRS, NIMBE, 91191, Gif-sur-Yvette, France. \\ ${ }^{\perp}$ Laboratory of Physical Chemistry of Electrolytes and Interfacial Nanosystems (PHENIX), \\ Sorbonne Université, CNRS, 75005 Paris, France
}

KEYWORDS: Clay, beidellite, polymer, polyethylene oxide, dextran, nematic, liquid-crystal, adsorption

ABSTRACT: Complex aqueous mixtures comprised of swelling clays and hydrosoluble polymers naturally occur in soils and play a major role in pedogenesis. They are also very often used for formulating oil-well drilling fluids, paints, and personal-care products. The suspensions of some natural clays, thanks to their large nanoparticle aspect ratio, spontaneously form nematic liquid-crystalline phases where the particles align parallel to each other, which affects their flow 
properties. We observed that adding small amounts of hydrosoluble polymers to these clay suspensions destabilizes the nematic phase with respect to the isotropic (disordered) phase. The polymers that we used (polyethylene oxide and dextran) were too small to adopt particlebridging conformations and small-angle X-ray scattering experiments showed that the structure of the nematic phase is not altered by polymer doping. However, the adsorption isotherm shows that the macromolecules adsorb onto the clay nanosheets, effectively coating them with a polymer layer. Our extension of Onsager's theory for polymer-coated platelets properly captures the experimental phase diagram and shows how the nematic phase destabilization can be due to the polymer adsorbing more on the platelet faces than at the rim. Because the flow properties of the nematic phase are very different from those of the isotropic phase, the presence or absence of the former phase is an important factor to be determined and considered to explain the rheological behavior of these complex systems. 


\section{INTRODUCTION}

Thanks to their low cost and wide availability, clay minerals find numerous industrial applications in very diverse areas such as cosmetics, paints, paper coatings, composites, and oildrilling fluids. Hydrosoluble polymers, like polyethylene oxide (PEO), are often added to aqueous clay formulations either to fine-tune their rheological properties for specific applications $^{1}$ or, more recently, to produce nanocomposite materials for pharmaceutical applications. ${ }^{2}$ Moreover, the interactions of dissolved organic matter with clays were also reported as playing an important role in soils where they control to a large extent water and nutrient retention capacity, and are also of prime importance in water treatment operations. ${ }^{3,4}$ Consequently, many fundamental studies have explored the structure and rheology of claypolymer aqueous mixtures over the last decades. Although several articles report on the investigation by neutron scattering of the adsorption of PEO on vermiculite, ${ }^{5-8}$ the vast majority of these studies focused on the laponite - PEO system, probably because laponite is a synthetic clay mineral that is well characterized and easy to purchase..$^{9-23}$ These articles described very puzzling rheological properties, such as sample rheo-thinning, shear-thickening, aging and rejuvenation, depending on the PEO concentration and molecular weight. A notable example is that of the "shake-gels" which are laponite/PEO fluid systems that become stiff enough, when

strongly sheared, to support their own weight, yet turn fluid again after several hours. ${ }^{24,25}$ Most of these original rheological properties were explained by the steric barrier against gelation due to the adsorption of PEO and, in the case of long enough PEO, by the presence of "bridging" conformations that give rise to a hybrid particle/polymer network.

However, laponite is singular by its small particle size $(D \approx 25 \mathrm{~nm})$ compared to natural clays $(D \approx 200-500 \mathrm{~nm})$ and its behavior may not be quite representative of that of swelling clay 
minerals. For example, aqueous suspensions of some natural clays, like beidellite and nontronite, spontaneously form a nematic liquid-crystal phase at low weight fraction (a few wt\%) whereas laponite suspensions undergo a sol/gel transition that prevents reaching the nematic phase at complete thermodynamic equilibrium. ${ }^{26-30}$ One reason for this difference in behavior is probably the huge aspect ratio of natural clay nanosheets $(D / t>300$, where $t=0.65 \mathrm{~nm}$ is the nanosheet thickness) compared to that of laponite $(\mathrm{D} / \mathrm{t}<40)$ since the onset of liquid-crystalline ordering generally takes place at lower concentrations for increasing particle diameter.

In this article, we report on the effect of PEO addition on the isotropic/nematic (i.e. disordered/ordered) phase transition of beidellite clay aqueous suspensions. Since this transition is of first-order, its most salient feature is the coexistence, in some clay concentration range, of two phases in the same sample: a nematic birefringent phase at the bottom and an isotropic phase at the top. Surprisingly, we observed that adding PEO to beidellite suspensions completely destabilizes the nematic phase with respect to the isotropic one. Since the adsorption of PEO on some other clays is well documented in the literature, ${ }^{12,15,31,32}$ we developed a theoretical model, based on the Onsager theory, that considers "dressed" clay nanosheets, coated with polymer, and we thus show that this unexpected experimental observation may be the sign that PEO adsorbs more onto the beidellite nanosheet faces than at its edge. 


\section{MATERIALS AND METHODS}

\section{Materials:}

The beidellite clay preparation procedure was already described in detail elsewhere ${ }^{28}$ and we therefore only describe it here briefly. Beidellite from Idaho was purchased from the Source Clays Repository at Purdue University, West Lafayette, Indiana, USA. $200 \mathrm{~g}$ of the as-received sample were ground in an agate mortar, dispersed in 5 liters of a $1 \mathrm{M} \mathrm{NaCl}$ solution and stirred for a few hours. Due to the high ionic strength in this suspension, once stirring was stopped, flocculated clay particles settled at the bottom of the vessel. The supernatant was then discarded and replaced by fresh $1 \mathrm{M} \mathrm{NaCl}$ solution. This operation was repeated three times, which ensures that all clay layers are exchanged with $\mathrm{Na}^{+}$counterions. The sample was then redispersed in 10 liters of deionized water, placed in various dialysis tubes (Visking 14000Da) and dialyzed against at least 25 liters of deionized water. The water was changed every 24 to 48 hours and the conductivity of the bath was checked. Dialysis was stopped when the conductivity was as low as $20 \mu \mathrm{S} / \mathrm{m}$. The suspension was then poured into Imhoff settling cones and, after 24 hours of sedimentation, the material located at the bottom of the cones was discarded. The final suspension was then subjected to successive centrifugation steps at 4600, 16000, and $34000 \mathrm{~g}$ (average values in the rotor). After each centrifugation step, the bottom fraction was collected and dispersed in deionized water to yield a free flowing suspension, whereas the supernatant was centrifuged at higher speed. Three size fractions were thus obtained. The one used in the present work is the last one; it has the smallest nanosheets, with an average equivalent particle diameter of $\mathrm{D}=200 \mathrm{~nm}$, as determined by transmission electron microscopy (TEM); the particle thickness is $\mathrm{t}=0.65 \mathrm{~nm}$. Moreover, TEM experiments showed that the beidellite particles have a rather irregular shape and a polydispersity distribution of about 30\% (Figure SI 1). Moreover, assuming 
that the clay sheets are fully delaminated in suspension, the specific surface area, $\mathrm{SSA}=770 \mathrm{~m}^{2} \cdot \mathrm{g}^{-1}$, can be estimated from the particle dimensions and density (2.7). The weight fraction of the beidellite suspension initial batch was determined by its weight loss upon drying to be $1.6 \mathrm{wt} \%$. The zeta potential of the clay particles in suspension is $-45 \mathrm{mV}$ and the particle charge density is $0.1 \mathrm{C} . \mathrm{m}^{-2}$. The $\mathrm{pH}$ of the stock clay suspension is 5.8 , which is probably due to the dissolution of atmospheric $\mathrm{CO}_{2}$. These aqueous suspensions of beidellite clay show very good colloidal stability over many years.

Most polymer / clay mixtures were prepared with PEO of molar mass $\mathrm{M}_{\mathrm{W}}=100 \mathrm{kDa}$, which corresponds to an average polymerization degree of 2300, purchased from Sigma-Aldrich. Additional experiments were also performed with PEO of $\mathrm{M}_{\mathrm{W}}=10 \mathrm{kDa}$ molar mass, as well as with dextran (a natural polysaccharide also purchased from Sigma-Aldrich) of molar mass $\mathrm{M}_{\mathrm{W}}=100 \mathrm{kDa}$. The polydispersity distributions of these polymers were not determined by the provider. The concentrations of the initial pure polymer solutions were defined by precisely weighing the constituents.

\section{Sample preparation:}

Colloidal mixtures of beidellite nanosheets and PEO were prepared by directly mixing the suspensions of nanosheets with solutions of PEO in deionized (18 M $2 . \mathrm{cm})$ water. The composition of each mixture was defined by the weight fractions, $\mathrm{C}_{\text {beid, }}$, and $\mathrm{C}_{\mathrm{PEO}}$, of the beidellite nanosheets, and PEO, respectively, to explore 2-dimensional phase diagrams. For this purpose, the proper volumes of nanosheet suspensions and PEO solutions were precisely measured with a calibrated micropipette and dilution lines, at constant $\mathrm{C}_{\text {beid }}$, or $\mathrm{C}_{\mathrm{PEO}}$, were prepared. The density of beidellite is 2.7 , so that the clay volume fraction, $\phi_{\text {beid, }}$, in the mixture is simply given by $\mathrm{C}_{\text {beid }} / 2.7$. The density of PEO is 1.1 and the volume fraction of PEO, $\phi_{\mathrm{PEO}}$, in the 
mixture is given by $\mathrm{C}_{\mathrm{PEO}} / 1.1$. The ionic strength of the mixtures is essentially set by the $\mathrm{NaCl}$ molarity $\left(10^{-4} \mathrm{M}\right)$ used in the last dialysis of the clay suspensions. All mixture samples had the same $1 \mathrm{~mL}$ volume and were poured into $2 \mathrm{~mL}$ glass vials equipped with tight Teflon-lined caps. All samples were stored in the dark.

\section{Visual observation of the samples:}

Series of samples, at constant $\mathrm{C}_{\text {beid }}$ or $\mathrm{C}_{\mathrm{PEO}}$, were examined with the naked eye, either in natural light or between crossed polarizers to assess the number of coexisting phases and to check for their birefringence. Photographs of the samples were recorded with an Olympus XZ-1 camera, at normal incidence. The samples were examined at different times after preparation, over a year, but most of the results shown here were obtained after about two months of sample aging.

\section{Small-angle X-ray scattering experiments:}

A series of small-angle X-ray scattering (SAXS) measurements of the beidellite / PEO system was made at the Swing SAXS beamline of the SOLEIL synchrotron facility at Saint-Aubin, France. The X-ray energy was $12 \mathrm{keV}(\lambda=0.1033 \mathrm{~nm})$, the sample-to-detector distance was $6.226 \mathrm{~m}$, so that the scattering vector modulus, $\mathrm{q}(\mathrm{q}=(4 \pi \sin \theta) / \lambda$ where $2 \theta$ is the scattering angle) ranged roughly between $10^{-2}$ and $1 \mathrm{~nm}^{-1}$. The beam size was $375 \times 75 \mu \mathrm{m}^{2}$ at the sample level, the scattering was recorded by an Eiger-4M detector, and the exposure time was typically $0.5 \mathrm{~s}$. The usual SAXS data reduction procedures were made, using the Swing data reduction software (Foxtrot 3.4.9), and the data was displayed either as 2-dimensional SAXS patterns or as plots of the scattered intensity versus scattering vector modulus, I(q), obtained by azimuthal averaging of the SAXS patterns. The samples were filled into cylindrical Lindemann glass capillaries of $1.0 \pm 0.1 \mathrm{~mm}$ diameter (Glas-Technik \& Konstruktion, Germany) and sealed with 
silicone glue. Each capillary tube was scanned along its vertical axis by recording the SAXS patterns every few millimetres.

\section{Adsorption isotherm:}

The adsorption isotherm of PEO on beidellite was obtained using the following protocol. A starting PEO solution at $20 \mathrm{~g} / \mathrm{L}$ was diluted to obtain a series of 9 solutions with concentrations ranging from 0 to $20 \mathrm{~g} / \mathrm{L}$. The viscosity of each of these solutions was then measured using an Anton Paar automatic Microviscosimeter AMVn, which yields a calibration curve relating viscosity with PEO concentration (Figure SI 2). $2 \mathrm{~mL}$ of each of these solutions were then mixed with $0.5 \mathrm{~mL}$ of a beidellite suspension of known weight fraction (typically around $15 \mathrm{~g} / \mathrm{L}$ ). After four hours, the suspensions were centrifuged at $105000 \mathrm{~g}$ to separate the liquid from the solid. The viscosity of the supernatant was measured and the content in PEO was deduced on the basis

of the calibration curve. The adsorbed amount was then measured by the rest method, i.e. $Q_{a}=\frac{\left(c_{i}-c_{e}\right) * V}{M}$ where $c_{i}$ and $c_{e}$ are the initial and equilibrium concentrations in PEO, respectively, $\mathrm{V}$ the suspension volume and $\mathrm{M}$ the mass of solid initially dispersed in the suspension. Moreover, several data points of the adsorption curve were checked by additional thermogravimetric analyses of the supernatant. 


\section{EXPERIMENTAL RESULTS AND DISCUSSION}

The addition of PEO to the suspensions of beidellite clay nanosheets did not result in any obvious effect such as flocculation or coacervation. In fact, detecting the effect of PEO doping on the samples required their observation between crossed polarizers. Under these conditions, the isotropic phase looks completely dark whereas the nematic phase, owing to its birefringence, looks bright. Consequently, samples belonging to the biphasic domain of the phase diagram, between the nematic and isotropic phases, show the isotropic/nematic phase coexistence in polarized-light, with the bright nematic phase residing at the bottom of the vial whereas the dark isotropic phase is found at the top. The isotropic phase has a concentration of clay nanosheets which is large enough to show strong flow-birefringence and Tyndall scattering. Indeed, we also checked, by measuring the weight loss upon drying, that the clay concentration in the isotropic phase is not much lower than that in the nematic phase, as expected from previous work. ${ }^{28}$

The observation of four series of samples of constant beidellite concentration and increasing PEO content, placed between crossed polarizers, reveals that the nematic phase is strongly destabilized with respect to the isotropic one (Figure 1). For example, in the series with $\mathrm{C}_{\text {beid }}=1.28 \mathrm{wt} \%$ (Figure $1 \mathrm{~b}$ ), the first sample, with no PEO, is almost completely nematic whereas in the last sample, with $\mathrm{C}_{\mathrm{PEO}}=1 \mathrm{wt} \%$, the nematic phase has almost completely vanished. In a similar way, in the series with $\mathrm{C}_{\text {beid }}=1.12 \mathrm{wt} \%$ (Figure 1c), the first sample is biphasic with almost equal proportions of nematic and isotropic phases whereas the sample, with $\mathrm{C}_{\mathrm{PEO}}=0.5 \mathrm{wt} \%$, has no nematic phase left. 


\section{a)}

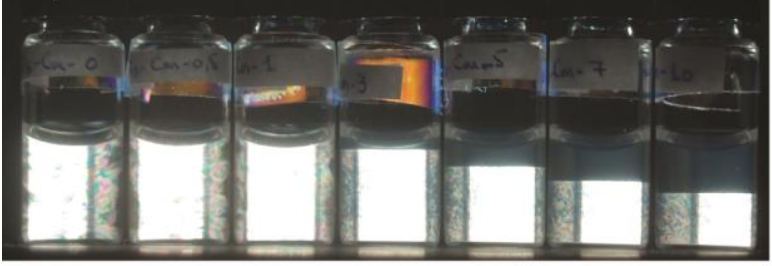
$\begin{array}{ll}0 & 0.05\end{array}$
$0.1 \quad 0.3$
0.5
$0.7 \quad 1$

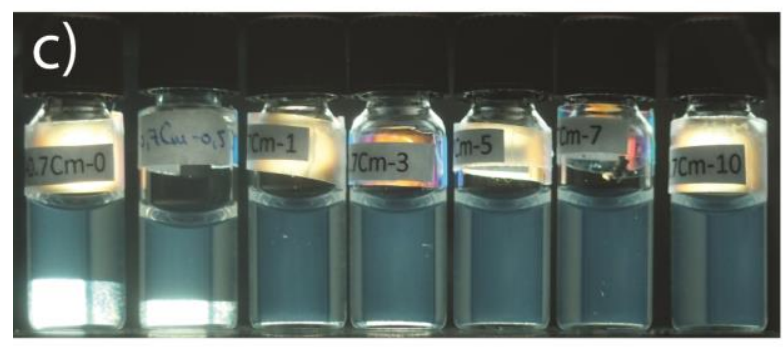

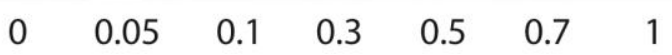

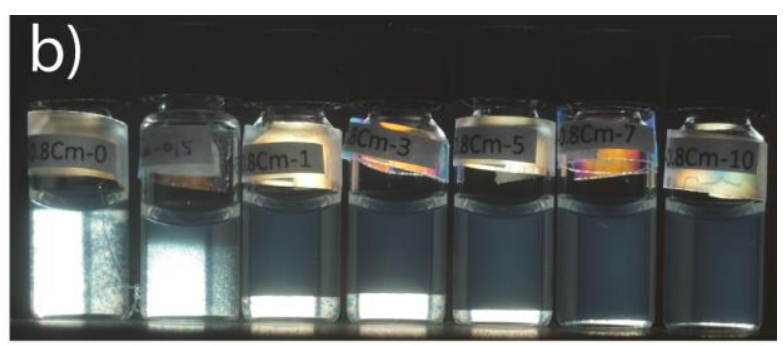

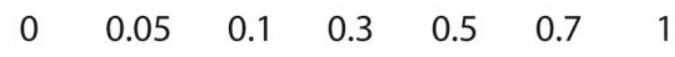

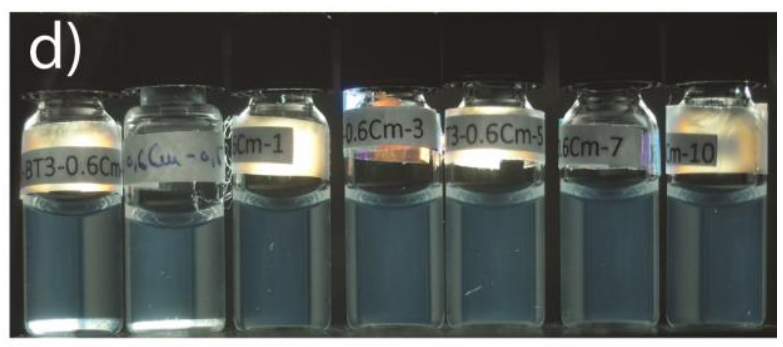

$\begin{array}{lllllll}0 & 0.05 & 0.1 & 0.3 & 0.5 & 0.7 & 1\end{array}$

Figure 1. Photographs of four series of sample tubes observed between crossed polarizers. For each series, the beidellite nanosheet weight fraction is kept constant: a) $\mathrm{C}_{\text {beid }}=1.6 \mathrm{wt} \%$, b) $\mathrm{C}_{\text {beid }}=1.28 \mathrm{wt} \%$, c) $\mathrm{C}_{\text {beid }}=1.12 \mathrm{wt} \%$, and d) $\mathrm{C}_{\text {beid }}=0.96 \mathrm{wt} \%$, but the $100 \mathrm{kDa}$ PEO weight fraction increases from left to right: $\mathrm{C}_{\mathrm{PEO}}=0,0.05,0.1,0.3,0.5,0.7$, and $1 \mathrm{wt} \%$. The nematic phase is clearly destabilized with respect to the isotropic phase by the addition of PEO.

Our synchrotron SAXS study of the beidellite / PEO system confirmed this phase identification (Figure 2) as the SAXS patterns were indeed those expected for the isotropic and nematic phases. The patterns of the nematic phase were anisotropic (Figure 2a) when the nematic part of the sample was aligned by the shear flow that occurred when the capillaries were filled. The anisotropy of the SAXS patterns demonstrates the long-range orientational order of the phase and, combined with the absence of any sharp scattering features, proves its nematic nature. ${ }^{29}$ In contrast, the SAXS patterns of the disordered phase (Figure $2 b$ ) only showed a strong isotropic halo and the corresponding azimuthally-averaged scattered intensity (see Materials and Methods section), I(q), was a featureless, monotonously decreasing, function of the scattering vector modulus q. 
In the nematic phase, I(q) shows marked scattering peaks. Moreover, in first approximation, since the particle form factor decays as $\mathrm{q}^{-2}$ in this q-range (called "intermediate" range), the quantity obtained by multiplying I(q) by $\mathrm{q}^{2}$ (Kratky representation) is proportional to the structure factor, $\mathrm{S}(\mathrm{q})$, of the phase (Figure $2 \mathrm{c}, \mathrm{d}) .{ }^{33} \mathrm{~S}(\mathrm{q})$ in the nematic phase has strong oscillations that classically arise from some liquid-like positional order of the nanosheets. In this system of negatively charged nanosheets that mostly interact through strong electrostatic repulsions, the average distance, $\mathrm{d}$, between nanosheets along the nematic director is related to the position, $\mathrm{q}_{0}$, of the first broad scattering peak by $d \approx 2 \pi / \mathrm{q}_{0}$. We therefore simply deduce the distance $\mathrm{d}$ from the peak position. Although $\mathrm{q}_{0}$ varies with the clay weight fraction, its dependence on PEO doping (Figure SI 3) did not display, within experimental accuracy, the typical large increase associated with the appearance of bridging conformations of the polymer. ${ }^{7}$ Note that this average distance between nanosheets is rather large, typically around $100 \mathrm{~nm}$, due to the very low $(<2 \mathrm{wt} \%)$ nanosheet weight fraction of the system. Moreover, we did not observe any systematic influence of PEO addition on the higher-order peaks, either in terms of peak intensity or width. 


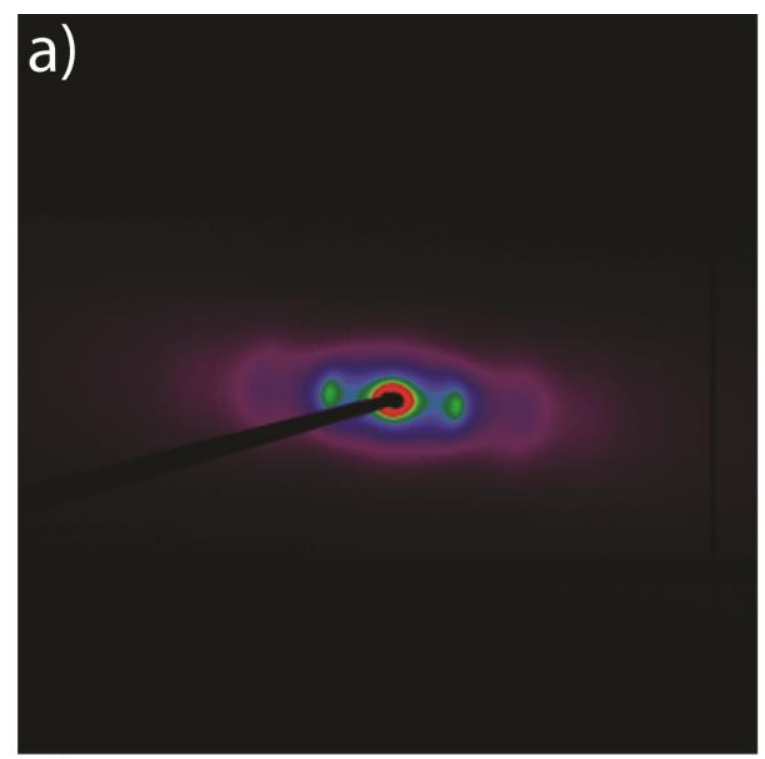

C)

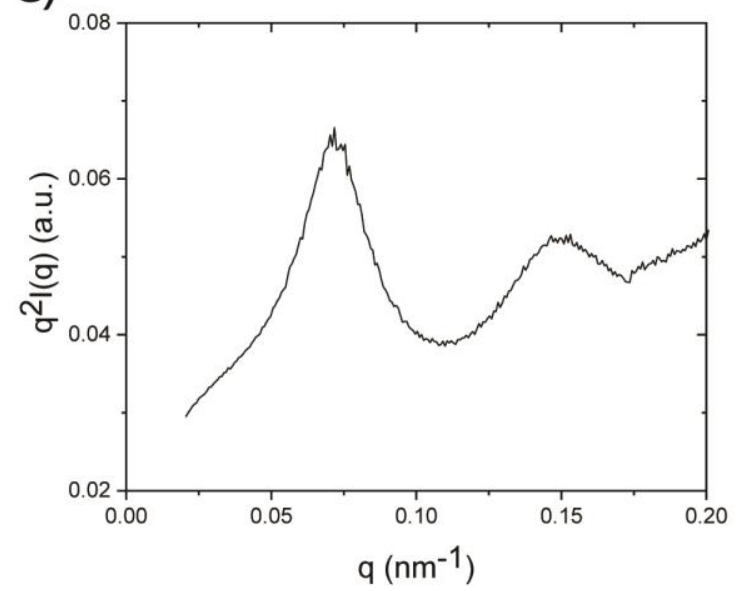

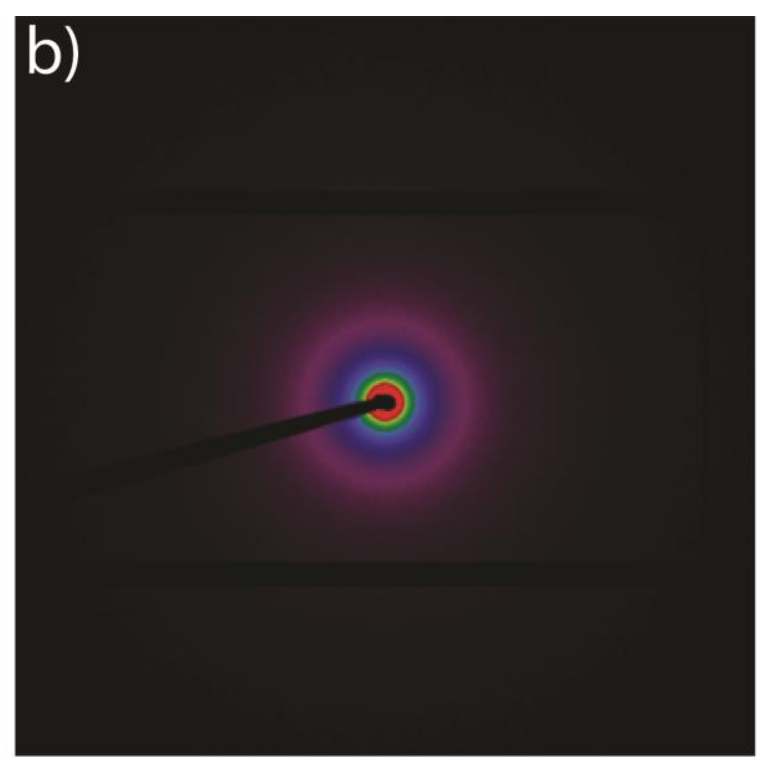

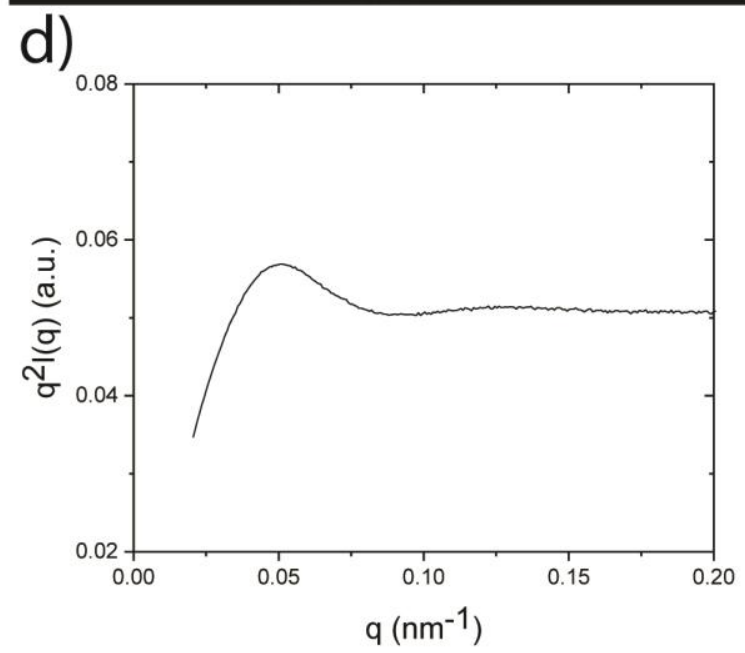

Figure 2. SAXS patterns of a) the nematic part and b) the isotropic part of the same biphasic beidellite / $100 \mathrm{kDa}$ PEO sample with $\mathrm{C}_{\text {beid }}=1.28 \mathrm{wt} \%$ and $\mathrm{C}_{\mathrm{PEO}}=0.05 \mathrm{wt} \%$ and $(\mathrm{c}, \mathrm{d})$ the corresponding curves of azimuthally-averaged scattered intensity in Kratky representation.

The phase diagram of the beidellite / PEO system, displayed in Figure 3, summarizes all our optical observations and SAXS results. In the sample point mesh, the borders of the isotropic/nematic biphasic region were approximately positioned close to the points representing samples that are almost entirely nematic or isotropic. The most salient feature of the phase 
diagram is that the biphasic region is very tilted towards large nanosheet concentrations. Then, this diagram shows how adding a small amount ( $\lesssim 1 \mathrm{wt} \%)$ of $100 \mathrm{kDa}$ PEO can completely destabilize the nematic phase with respect to the isotropic one.

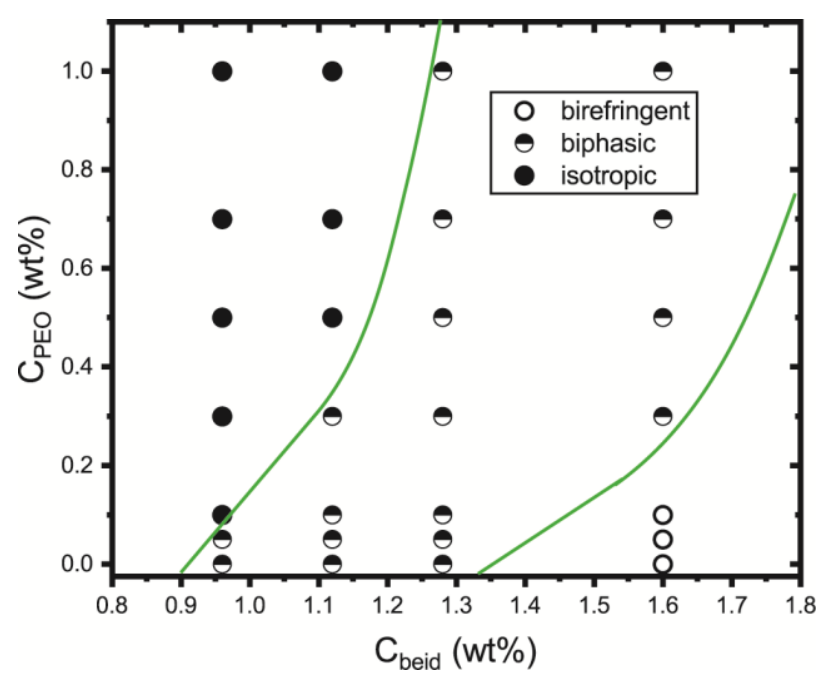

Figure 3. Experimental phase diagram of the PEO $(100 \mathrm{kDa}) /$ beidellite colloidal suspensions. The green lines show the boundaries of the isotropic/nematic coexistence region.

Our investigation of PEO adsorption onto beidellite nanosheets shows that this polymer has large affinity for the clay, as illustrated by the adsorption isotherm (Figure 4). Indeed, an initial sharp adsorption stage is clearly observed, followed by a saturation plateau at a value of $\approx 0.7 \mathrm{~g}$ adsorbed PEO per g of clay. This result corresponds to $0.9 \mathrm{mg} / \mathrm{m}^{2}$, a value quite comparable to those previously reported $\left(0.6-0.9 \mathrm{mg} / \mathrm{m}^{2}\right.$, depending on the polymer molecular weight $)$ for the adsorption of PEO on laponite. ${ }^{12}$ The series of samples shown in Figure 1 all belong to the regime of initial steep adsorption rise in Figure 4. Our adsorption measurements, in line with previous reports on laponite, ${ }^{9,11,12}$ means that the PEO also adsorbs on beidellite, with a very similar adsorption isotherm. Lal et al and De Lisi et al who used neutron scattering techniques reported that both short and long PEO chains adsorbed on laponite form a thin layer of only a 
few nanometers thickness. ${ }^{9,17}$ Burchill et al reached a similar conclusion for short PEO chains adsorbed on Na-montmorillonite. ${ }^{34}$ Then, the classical depletion interactions ${ }^{35}$ are probably irrelevant for this hybrid system, at least for $\mathrm{C}_{\mathrm{PEO}}<0.5 \mathrm{wt} \%$ where most of the destabilization of the nematic phase takes place. Moreover, the principal signature of depletion interactions on the phase diagram is to widen the biphasic gap, not to tilt it towards higher particle concentration. ${ }^{36}$

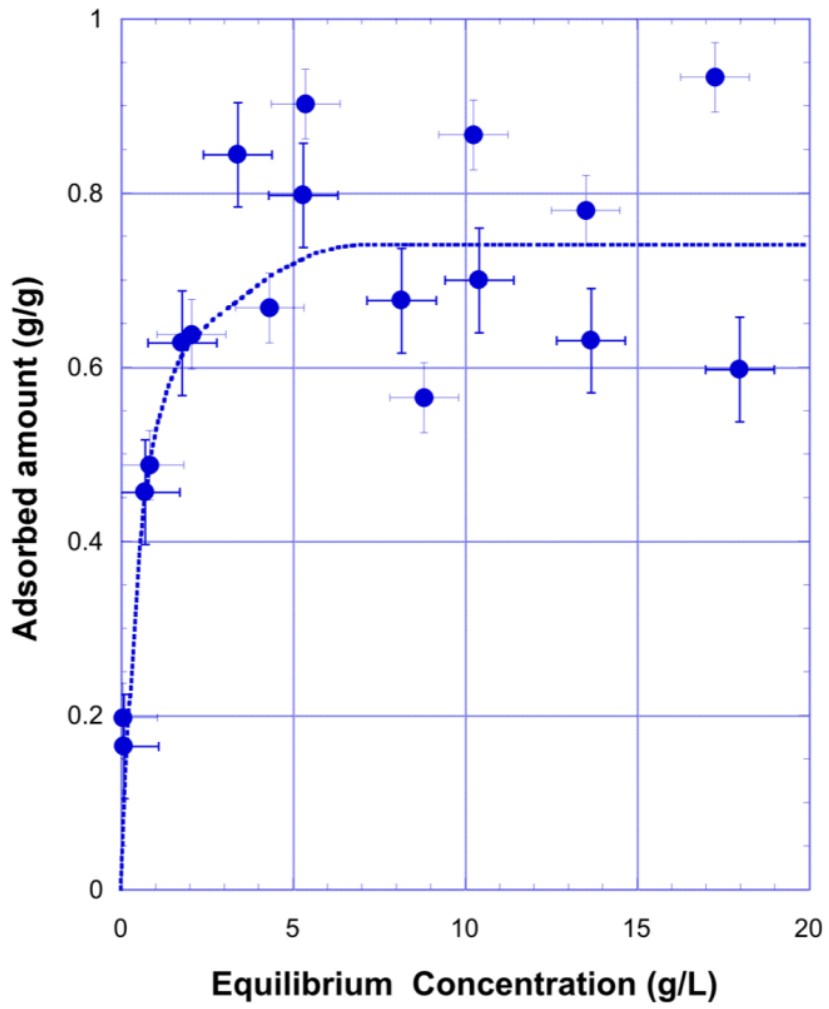

Figure 4. Adsorption isotherm of PEO (100 kDa) on beidellite, showing the adsorbed mass of PEO per gram of beidellite versus the PEO concentration in the solution. (The dotted line is just a guide to the eye.) 
The values found in the literature ${ }^{37,38}$ for the gyration radius of free PEO in water at room temperature are somewhat scattered but a value of $\mathrm{R}_{\mathrm{g}} \approx 25 \mathrm{~nm}$ seems reasonable for PEO with $100 \mathrm{kDa}$ molar mass. In principle, the partially adsorbed PEO coils should show a succession of loops, adsorbed trains, and dangling tails, or they might even "bridge" neighboring cofacial nanosheets. ${ }^{39}$ The value of $0.7 \mathrm{~g}$ adsorbed PEO per $\mathrm{g}$ of clay corresponds to about 230 PEO coils per beidellite nanosheet (115 on each side), which is roughly six times the full particle coverage if the PEO coils adsorbed in random-coil conformation, which rules out this possibility. Moreover, bridging conformations seem quite improbable because the average distance between nanosheets is at least $80 \mathrm{~nm}$ for all samples, and therefore much larger than the PEO end-to-end distance, resulting in an elastic energy penalty for stretching $\approx \mathrm{k}_{\mathrm{B}} \mathrm{T}\left(\mathrm{d} / \mathrm{R}_{\mathrm{g}}\right)^{2}>10 \mathrm{k}_{\mathrm{B}} \mathrm{T}^{40}$ This is consistent with the absence of any sign of gelation or polymer bridging flocculation in our samples. Therefore, in the following theoretical model (see next section), we consider that the PEO macromolecules adsorb as a succession of trains, loops, and tails, giving rise to a polymer coating layer on the beidellite nanosheets.

This assumption is further confirmed by our observation that the nematic phase of the beidellite suspensions is also destabilized (see Figure SI 4-6) by adding roughly the same amount of PEO of $\mathrm{M}_{\mathrm{w}} 10 \mathrm{kDa}$ as with PEO of $\mathrm{M}_{\mathrm{w}} 100 \mathrm{kDa}$, which means that the total PEO length is not a relevant parameter (at least in the range that we explored). Note also that PEO does not bear any electric charge at neutral $\mathrm{pH}$ and therefore should not alter, at such low concentrations, the electrostatic repulsions between nanosheets.

We also observed the destabilization of the nematic phase of beidellite clay suspensions using dextran instead of PEO (See Figure SI 7). Although both polymers are hydrosoluble, the molecular structure of dextran is quite different from that of PEO because the former is a 
polysaccharide. Moreover, similar experiments done with nematic suspensions of nontronite clay $^{27}$ showed the same effect (See Figure SI 8). Therefore, the disappearance of the nematic phase upon addition of adsorbing polymer seems to be a rather general phenomenon, possibly amenable to a generic theoretical description.

\section{THEORY OF ORDERING TRANSITIONS IN FLUIDS OF POLYMER-COATED NANOSHEETS}

\section{SECOND-VIRIAL THEORY FOR POLYMER-COATED PLATELETS:}

Inspired by a previously reported model for polymer-coated rods $^{41}$ we consider a hard cylindrical platelet of thickness $L$ and diameter $D$ enveloped by a uniform layer of adsorbed polymer of thickness $\delta$. Near-field electrostatic interactions between the platelets, which we shall not consider in further detail, may be incorporated through an effective platelet thickness that is larger than the bare hard-core value. While multi-particle correlations unmistakably play a role in the thermodynamics of discotic fluids, ${ }^{42,43}$ we restrict ourselves to considering interactions between particle pairs alone, in the supposition that such a second-virial approximation provides valuable qualitative insight into the ordering properties of nanosheets. The second virial coefficient between two such platelets at random orientation indicated by the enclosed angle between their normals $\gamma$ reads:

$$
B_{2}(\gamma)=-\frac{1}{2} \int d r[\exp (-\beta U(r ; \gamma))-1]
$$

with $\beta^{1}=k_{B} T$ the thermal energy in terms of Boltzmann's constant $k_{B}$ and temperature $T, U$ the pair potential and $\mathbf{r}$ the distance between the centres-of-mass of the platelets. A simple 
approximation is to assume the pair interaction to be infinitely repulsive when the hard-cores overlap, but of finite repulsion $\varepsilon>0$ when the polymer layer of one particle overlaps with either the layer or hard-core of the other particle. Since the repulsive potential is constant within the cylindrical envelope of the disc, the second-virial coefficient is simply proportional to the excluded volume $\mathrm{v}_{\mathrm{ex}}$ (details can be found in Reference ${ }^{44}$ ), so that we write for the coated discs:

$$
B_{2}(\gamma)=-\frac{1}{2}\left[\left(e^{-\beta \varepsilon}-1\right) v_{e x}\left(L_{\delta}, D_{\delta}\right)-e^{-\beta \varepsilon} v_{e x}(L, D)\right]
$$

with $L_{\delta}=L+2 \delta$ the total thickness of the platelet (hard-core plus polymer coating) and, similarly, $D_{\delta}=D+2 \xi \delta$ for the diameter. Here, $\xi$ is a measure for the amount of edge adsorption compared to face adsorption, so that $\xi=0$ refers to no edge adsorption, $\xi=1$ to equal adsorption at the face and edge, while $\xi>1$ corresponds to preferential adsorption at the plate edge. The key quantity featuring in $B_{2}$ is the orientation-dependent excluded volume, defined as: ${ }^{45}$

$$
v_{e x}(L, D)=\frac{\pi}{2} D^{3}|\sin \gamma|+L D^{2}\left(\frac{\pi}{2}+2 E(\gamma)+\frac{\pi}{2}|\cos \gamma|\right)+2 L^{2} D|\sin \gamma|
$$

with $E(\gamma)$ an elliptic integral of the second kind. Unlike rods, ${ }^{41}$ the excluded volume of platelets, at least up to leading order for flat particles, does not depend on the thickness $L$ which only enters through higher-order correction terms that depend quite subtly on the inter-particle orientation. This will likely impact the phase diagram in ways that are qualitatively different from that of polymer-coated rods. ${ }^{41}$

\section{ISOTROPIC-NEMATIC COEXISTENCE:}

The total Helmholtz free energy $F$ of a platelet fluid composed of $N$ particles reflects a balance between orientational entropy and pair interactions encoded in the second-virial coefficient: ${ }^{44,45}$ 


$$
\frac{\beta F}{N} \sim \ln \rho-1+\langle\ln 4 \pi f\rangle_{f}+\rho\left\langle\left\langle B_{2}(\gamma)\right\rangle\right\rangle_{f}
$$

where the brackets denote an average with respect to the orientational distribution function $f$ which is a priori unknown. The chemical potential and pressure follow from the free energy using standard thermodynamic derivatives and read:

$$
\begin{aligned}
& \beta \mu \sim \ln \rho+\langle\ln 4 \pi f\rangle_{f}+2 \rho\left\langle\left\langle B_{2}\right\rangle\right\rangle_{f} \\
& \beta P \sim \rho+\rho^{2}\left\langle\left\langle B_{2}\right\rangle\right\rangle_{f}
\end{aligned}
$$

A simple Gaussian approximation for $f$ enables us to render the free energy in algebraic form. ${ }^{44}$ Indeed, up to leading order we find for strongly aligned nematics and for isotropic order, respectively: ${ }^{46}$

$$
\begin{aligned}
\langle\ln 4 \pi f\rangle_{f} & \sim \ln \alpha-1 \quad(N) \\
\langle\ln 4 \pi f\rangle_{f} \sim 0 \quad & \quad(I)
\end{aligned}
$$

with $\alpha$ a measure for the nematic order. Similarly, we find for the angular contributions in $B_{2}$

$$
\begin{gathered}
\langle\langle|\sin \gamma|\rangle\rangle_{f} \sim \sqrt{\frac{\pi}{\alpha}} \quad(N) \\
\langle\langle|\sin \gamma|\rangle\rangle_{f} \sim \frac{\pi}{4} \quad(I)
\end{gathered}
$$

and:

$$
\begin{array}{r}
\langle\langle E(\gamma)\rangle\rangle_{f} \sim \frac{\pi}{2} \\
\langle\langle E(\gamma)\rangle\rangle_{f} \sim \frac{\pi^{2}}{8} \quad(I)
\end{array}
$$

and finally: 


$$
\begin{array}{r}
\langle\langle|\cos \gamma|\rangle\rangle_{f} \sim 1 \\
\langle\langle|\cos \gamma|\rangle\rangle_{f} \sim \frac{1}{2} \quad(I)
\end{array}
$$

Minimizing the free energy for $\alpha$ we find a simple quadratic relationship between the degree of nematic order and the plate concentration $\rho=N / V$ :

$$
\alpha \sim \frac{\pi}{4} \rho^{2} \alpha_{0}^{2}
$$

with

$$
\alpha_{0} \sim \frac{1}{2}\left[\left(1-e^{-\beta \varepsilon}\right)\left(\frac{\pi}{2} D_{\delta}^{3}+2 L_{\delta}^{2} D_{\delta}\right)+e^{-\beta \varepsilon}\left(\frac{\pi}{2} D^{3}+2 L^{2} D\right)\right]
$$

In what follows we will use $c=\left(\pi^{2} / 16\right) \rho D^{3}$ as a dimensionless measure for the plate concentration which yields the well-known asymptotic values for the coexistence concentrations, $c_{I}=3.45$ and $c_{N}=5.12$ for strongly anisotropic particles in the limit of zero thickness $L / D \rightarrow 0 .{ }^{44}$ Next, we explore the effect of an increasing thickness $\delta$ of adsorbed polymer, induced by an increasing amount of polymer added to the system, onto the isotropic-nematic phase diagram. For simplicity, we will set the hard-core aspect-ratio to zero $(L / D=0)$ and we further assume that increasing the amount of added polymer does not markedly change the soft repulsion $\varepsilon \sim 1 / \mathrm{k}_{\mathrm{B}} \mathrm{T}$ between overlapping polymer coatings. Figure 5 showcases the subtle effect of edge adsorption on the phase behavior; while strong edge adsorption $(\xi=1)$ favors nematic order, a marked weakening or absence of edge adsorption $(\xi=0)$ clearly stabilizes isotropic order, as observed in the experiment. 


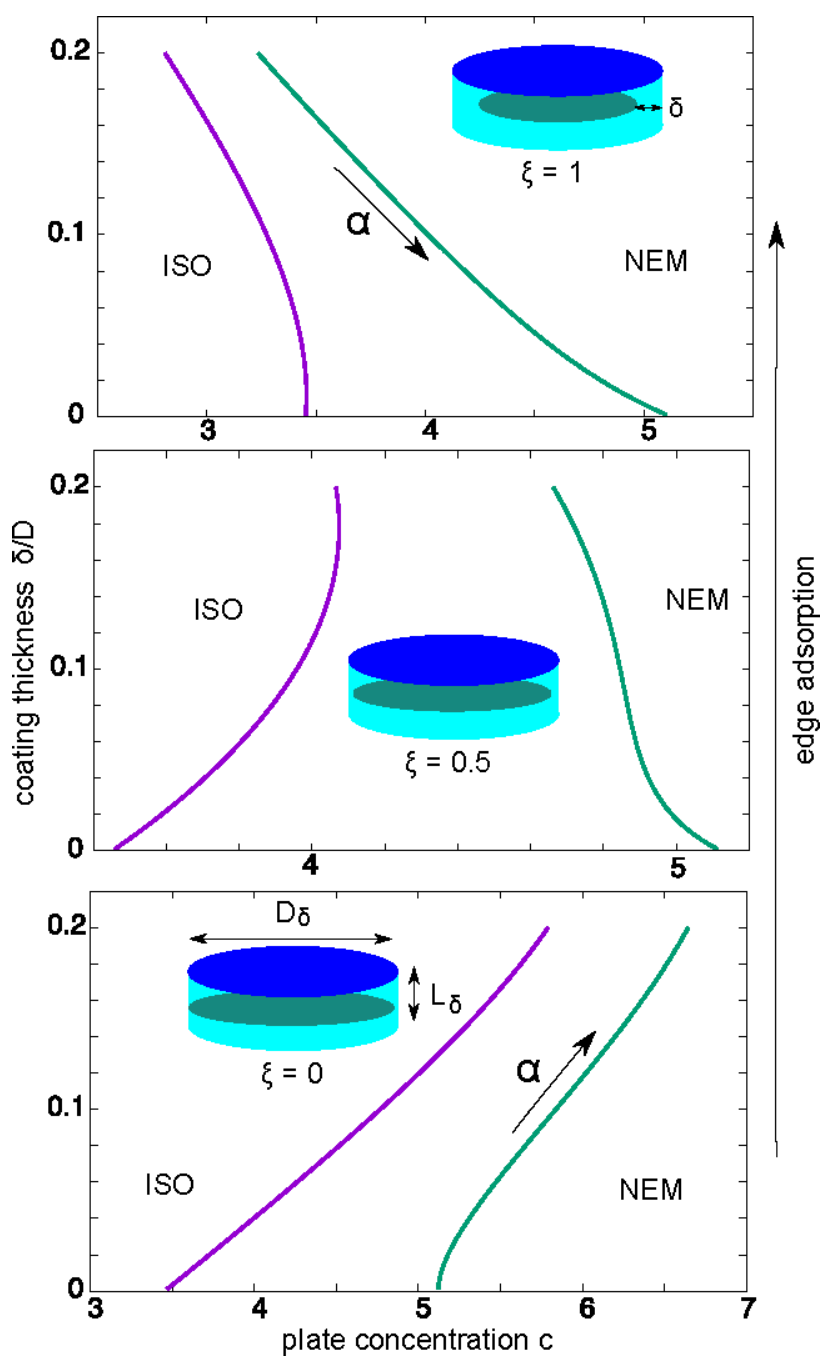

Figure 5. Isotropic-nematic phase behavior for polymer-coated platelets at different degrees of edge adsorption $\xi$. The hard-core aspect ratio is $L / D=0$ and the coating repulsion is $\varepsilon=1 / k_{B} T$. These scenarios are robust against changing $\varepsilon>0$ and/or $L / D$. The direction of change in nematic order $\alpha$ at coexistence is indicated by the arrows.

One could also envisage a scenario where adsorption produces a coating of fixed size $\delta$ which becomes increasingly dense in polymer, thus leading to enhanced repulsion $\varepsilon$ as more polymers adsorb. We find that keeping $\delta=0.1$ and increasing $\varepsilon$ produces scenarios that are qualitatively similar to the ones in Figure 5. 
We reiterate that the model presented here does not claim to give quantitative predictions for the experimental systems studied. In fact, comparing the experimental and theoretical phase diagrams, we find that the experimental concentrations are typically a factor 4 lower than the theoretical ones (even in the absence of a polymer layer). We attribute this discrepancy to the intricate effects of far-field electrostatic forces acting between pairs, triplets, etc. of discs, ${ }^{47}$ which to this day remain largely elusive and therefore cannot straightforwardly be incorporated into the theoretical model.

We further mention that the current predictions could be qualitatively affected by diameter and thickness dispersity of the discs as present in the experimental situation. In principle, this could be scrutinized via a straightforward extension of the present second-virial model by allowing the disc dimensions to follow a continuous distribution (e.g. of the log-normal form) with a prescribed standard deviation. $^{48}$

\section{EFFECT OF LAYER COMPRESSION}

Reference ${ }^{41}$ provides a simple route to addressing the effect of polymer compression on the fluid phase behavior. The central assumption is that compression of the polymer layers due to crowding of neighboring platelets induces an entropic penalty but at the same time reduces the excluded volume of the platelets. A compression factor $0<\gamma_{c}<1$ expresses the degree of compression. The free energy penalty associated with this reads:

$$
\frac{\beta F}{N} \sim \frac{k}{2}\left(\gamma_{c}^{2}+\gamma_{c}^{-2}-2\right)
$$


which reduces to zero for the uncompressed case $\left(\gamma_{c}=1\right)$ as it should. Further, $k>0$ is a rigidity constant reflecting the "softness" or elasticity of the polymer layer. It should be roughly proportional to the amount of polymer adsorbed onto the platelet. Intuitively, the local polymer concentration within the adsorbed layer should also determine the layer-to-layer repulsion strength $\varepsilon$, so we expect these two energy scales to be intimately linked. The effective dimensions of the coated platelet become:

$$
L_{\delta}=L+2 \xi_{c} \delta
$$

$$
D_{\delta}=D+2 \gamma_{c} \delta
$$

The trade-off between the entropic penalty due to overlapping polymers and reduction of excluded volume results in an equilibrium compression which lies somewhere between full compression $\left(\gamma_{c}=0\right)$ and no compression at all $\left(\gamma_{c}=1\right)$. Minimization of the total free energy, including Eq. (12) with respect to $\gamma_{c}$ yields:

$$
k\left(\gamma_{c}-\gamma_{c}^{-3}\right)+\rho \frac{\partial\left\langle\left\langle B_{2}(\gamma)\right\rangle\right\rangle}{\partial \gamma_{c}}=0
$$

This condition, which cannot be resolved analytically, gives the equilibrium value for the compression factor for a given particle concentration and rigidity constant $k$. Note that the compression will generally be different for the isotropic and nematic phase. Results are shown in Figure 6. Even though the degree of compression is very small $\left(\gamma_{c}=0.995\right.$ at most for both phases) the change in phase behavior is quite dramatic, with a stable reentrant feature emerging at low polymer addition in the absence of edge adsorption $(\xi=0)$. 

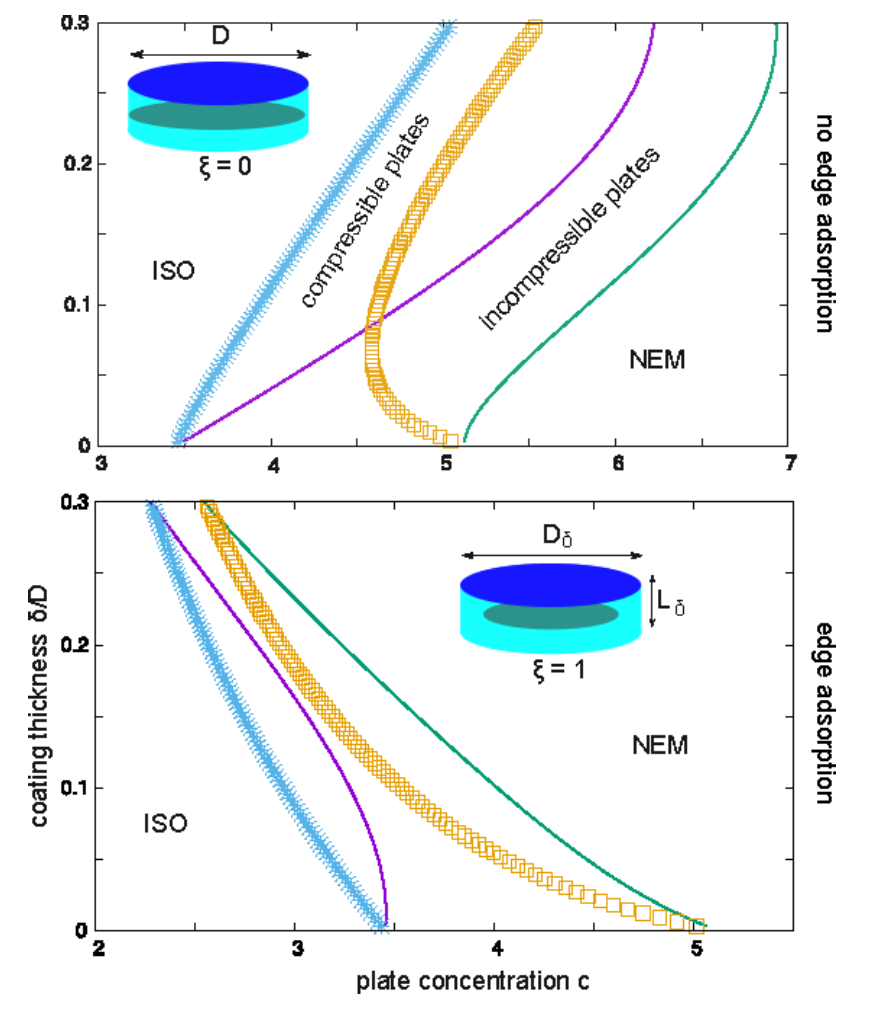

Figure 6. I-N phase diagram of compressible platelets with $k=100$. For the no-edge adsorption case $(\xi=0)$, a re-entrant effect emerges around $\delta / D=0.05$.

The model for compressible plates demonstrates that even the tiniest amount of polymer compression (about $5 \%$ ) leads to significant deviations in the isotropic-nematic phase behavior compared to fully incompressible discs. Despite the dilute nature of the systems considered in the experiment, direct disc-disc collisions must be frequent enough to generate nematic order. Each collision event is affected by the compressibility of the adsorbed polymer layers, even for extremely rigid polymer layers (say with $\mathrm{k}>>100$ ).

\section{CONCLUSIONS}

As expected for systems of highly anisotropic plate-like nanoparticles, beidellite clay aqueous suspensions spontaneously show nematic order. However, doping these suspensions with small 
amounts of adsorbing hydrosoluble polymer, with gyration radius much smaller than the average distance between particles, destabilizes their nematic order. Our SAXS experiments showed that polymer doping does not affect the structure of the nematic phase but only weakens its thermodynamic stability. According to our theoretical model, this phenomenon can be explained by the selective adsorption of the polymer on the faces of the nanosheets rather than at their edge. Previous neutron scattering and NMR studies of PEO adsorbed on laponite had inferred that a large number of polymer chains were "wrapped" around the laponite rim. ${ }^{11}$ However, in that study, the gyration radius of the PEO was about equal to the particle size whereas it is ten times smaller than the particle size in the present case. Moreover, neutron scattering techniques are less sensitive to the loops and dangling chains which are not adsorbed on the particles but nevertheless take part of the soft repulsion between the particles. Our model, based on Onsager's theory, shows that this additional soft repulsion may indeed bring about the destabilization of the nematic phase if polymer adsorption is stronger on the faces than on the edge of the clay nanosheet. This explanation is in good agreement with previous reports showing that PEO chains adsorb on the clay faces through complexation with the interlayer charge compensating cations. ${ }^{49-51}$ The destabilization of the nematic phase through polymer adsorption seems a fairly general phenomenon since we observed it for a wide range of PEO molecular weight, provided that there are no bridging conformations, not only with PEO but also with dextran, and also with nematic suspensions of nontronite clay. Furthermore, we found that the molar mass of the polymer is not a much relevant parameter, in the range that we explored, as the destabilization of the nematic phase seemed independent of the molar mass. From the point of view of applications, this work is relevant to most situations involving flow of clay / polymer systems because nematic liquid crystals have hydrodynamic properties (anisotropic viscosity, orientation- 
dependent shear-thinning ...) very different from those of usual isotropic liquids. ${ }^{52}$ This means that the alignment state of these systems should be carefully monitored in such situations to help interpret unexpected rheological behaviors. 


\section{ASSOCIATED CONTENT}

\section{Supporting Information.}

Figure SI 1: Transmission electron microscopy image of the beidellite nanosheets.

Figure SI 2: Calibration graphs of the PEO concentration versus the suspension viscosity for the $10 \mathrm{kDa}$ and $100 \mathrm{kDa}$ PEO.

Figure SI 3: Graph showing the average distance, d, between nanosheets in the nematic phase versus PEO concentration.

Figure SI 4: Photographs of series of sample tubes showing that the nematic phase of beidellite suspensions is destabilized with respect to the isotropic phase by the addition of PEO of $\mathrm{M}_{\mathrm{w}}$ $10 \mathrm{kDa}$.

Figure SI 5: Phase diagram of the (10 kDa) PEO / beidellite colloidal suspensions.

Figure SI 6: Adsorption isotherm of PEO (10 kDa) on beidellite.

Figure SI 7: Photographs of series of sample tubes showing that the nematic phase is destabilized with respect to the isotropic phase by the addition of dextran of $\mathrm{M}_{\mathrm{w}} 100 \mathrm{kD}$.

Figure SI 8: Photographs of series of sample tubes showing that the nematic phase of nontronite suspensions is destabilized with respect to the isotropic phase by the addition of PEO of $\mathrm{M}_{\mathrm{w}} 100 \mathrm{kDa}$.

\section{ACKNOWLEDGMENT}

We would like to thank Henk Lekkerkerker and Erwan Paineau for very helpful discussions, Emmanuel Beaudoin for preliminary experiments, Lina Cherni for thermogravimetric 
experiments, Claire Goldmann for help with the sample preparation, and Doru Constantin for help with the SAXS experiments. We acknowledge SOLEIL for provision of synchrotron radiation facilities (under the approved proposal \# 20181283). The Foxtrot 3.4.9 software was developed by G.Viguier, R.Girardot, J.Perez, M.Ounsy, and the Xenocs Company. JCG, KER, LM, and PD would like to thank the "Agence Nationale de la Recherche" for financial support, grant nANR-17-CE04-0003, project 4WATER. 


\section{REFERENCES}

(1) Haraguchi, K.; Takehisa, T. Nanocomposite Hydrogels: A Unique Organic-Inorganic Network Structure with Extraordinary Mechanical, Optical, and Swelling/De-Swelling Properties. Advanced Materials 2002, 14 (16), 1120-1124. https://doi.org/10.1002/15214095(20020816)14:16<1120::AID-ADMA1120>3.0.CO;2-9.

(2) Schexnailder, P.; Schmidt, G. Nanocomposite Polymer Hydrogels. Colloid Polym. Sci. 2009, 287 (1), 1-11. https://doi.org/10.1007/s00396-008-1949-0.

(3) Kalbitz, K.; Solinger, S.; Park, J. H.; Michalzik, B.; Matzner, E. Controls on the Dynamics of Dissolved Organic Matter in Soils: A Review. Soil Sci. 2000, 165 (4), 277-304. https://doi.org/10.1097/00010694-200004000-00001.

(4) Arias-Estevez, M.; Lopez-Periago, E.; Martinez-Carballo, E.; Simal-Gandara, J.; Mejuto, J.-C.; Garcia-Rio, L. The Mobility and Degradation of Pesticides in Soils and the Pollution of Groundwater Resources. Agric. Ecosyst. Environ. 2008, 123 (4), 247-260. https://doi.org/10.1016/j.agee.2007.07.011.

(5) Smalley, M. V.; Hatharasinghe, H. L. M.; Osborne, I.; Swenson, J.; King, S. M. Bridging Flocculation in Vermiculite-PEO Mixtures. Langmuir 2001, 17 (13), 3800-3812. https://doi.org/10.1021/la0008232.

(6) Swenson, J.; Smalley, M. V.; Hatharasinghe, H. L. M. Structure of Bridging Polymers. J. Chem. Phys. 1999, 110 (19), 9750-9756. https://doi.org/10.1063/1.478940.

(7) Swenson, J.; Smalley, M. V.; Hatharasinghe, H. L. M. Mechanism and Strength of Polymer Bridging Flocculation. Phys. Rev. Lett. 1998, 81 (26), 5840-5843. https://doi.org/10.1103/PhysRevLett.81.5840.

(8) Swenson, J.; Smalley, M. V.; Hatharasinghe, H. L. M.; Fragneto, G. Interlayer Structure of a Clay-Polymer-Salt-Water System. Langmuir 2001, 17 (13), 3813-3818. https://doi.org/10.1021/la001353e.

(9) Lal, J.; Auvray, L. Interaction of Polymer with Clays. J. Appl. Crystallogr. 2000, 33 (1), 673-676. https://doi.org/10.1107/S0021889899013308.

(10) Nelson, A.; Cosgrove, T. Dynamic Light Scattering Studies of Poly(Ethylene Oxide) Adsorbed on Laponite: Layer Conformation and Its Effect on Particle Stability. Langmuir 2004, 20 (24), 10382-10388. https://doi.org/10.1021/la049323p.

(11) Nelson, A.; Cosgrove, T. A Small-Angle Neutron Scattering Study of Adsorbed Poly(Ethylene Oxide) on Laponite. Langmuir 2004, 20 (6), 2298-2304. https://doi.org/10.1021/la035268t.

(12) Mongondry, P.; Nicolai, T.; Tassin, J. F. Influence of Pyrophosphate or Polyethylene Oxide on the Aggregation and Gelation of Aqueous Laponite Dispersions. J. Colloid Interface Sci. 2004, 275 (1), 191-196. https://doi.org/10.1016/j.jcis.2004.01.037.

(13) Loizou, E.; Butler, P.; Porcar, L.; Kesselman, E.; Talmon, Y.; Dundigalla, A.; Schmidt, G. Large Scale Structures in Nanocomposite Hydrogels. Macromolecules 2005, 38 (6), 20472049. https://doi.org/10.1021/ma047411a.

(14) Baghdadi, H. A.; Sardinha, H.; Bhatia, S. R. Rheology and Gelation Kinetics in Laponite Dispersions Containing Poly(Ethylene Oxide). J. Polym. Sci. Pt. B-Polym. Phys. 2005, 43 (2), 233-240. https://doi.org/10.1002/polb.20317.

(15) De Lisi, R.; Lazzara, G.; Lombardo, R.; Milioto, S.; Muratore, N.; Liveri, M. L. T. Adsorption of Triblock Copolymers and Their Homopolymers at Laponite Clay/Solution 
Interface. Role Played by the Copolymer Nature. Phys. Chem. Chem. Phys. 2005, 7 (23), 3994-4001. https://doi.org/10.1039/b510891h.

(16) Baghdadi, H. A.; Jensen, E. C.; Easwar, N.; Bhatia, S. R. Evidence for Re-Entrant Behavior in Laponite-PEO Systems. Rheol. Acta 2008, 47 (2), 121-127. https://doi.org/10.1007/s00397-007-0218-3.

(17) De Lisi, R.; Gradzielski, M.; Lazzara, G.; Milioto, S.; Muratore, N.; Prevost, S. Aqueous Laponite Clay Dispersions in the Presence of Poly(Ethylene Oxide) or Poly(Propylene Oxide) Oligomers and Their Triblock Copolymers. J. Phys. Chem. B 2008, 112 (31), 9328-9336. https://doi.org/10.1021/jp8024073.

(18) Schexnailder, P.; Loizou, E.; Porcar, L.; Butler, P.; Schmidt, G. Heterogeneity in Nanocomposite Hydrogels from Poly(Ethylene Oxide) Cross-Linked with Silicate Nanoparticles. Phys. Chem. Chem. Phys. 2009, 11 (15), 2760-2766. https://doi.org/10.1039/b820452g.

(19) Takeda, M.; Matsunaga, T.; Nishida, T.; Endo, H.; Takahashi, T.; Shibayama, M. RheoSANS Studies on Shear Thickening in Clay-Poly(Ethylene Oxide) Mixed Solutions. Macromolecules 2010, 43 (18), 7793-7799. https://doi.org/10.1021/ma101319j.

(20) Fall, A.; Bonn, D. Shear Thickening of Laponite Suspensions with Poly(Ethylene Oxide). Soft Matter 2012, 8 (17), 4645-4651. https://doi.org/10.1039/c2sm07089h.

(21) Lorthioir, C.; Khalil, M.; Wintgens, V.; Amiel, C. Segmental Motions of Poly(Ethylene Glycol) Chains Adsorbed on Laponite Platelets in Clay-Based Hydrogels: A-NMR Investigation. Langmuir 2012, 28 (20), 7859-7871. https://doi.org/10.1021/la3010757.

(22) Zulian, L.; Marques, F. A. de M.; Emilitri, E.; Ruocco, G.; Ruzicka, B. Dual Aging Behaviour in a Clay-Polymer Dispersion. Soft Matter 2014, 10 (25), 4513-4521. https://doi.org/10.1039/c4sm00172a.

(23) Zulian, L.; Ruzicka, B.; Ruocco, G. Influence of an Adsorbing Polymer on the Aging Dynamics of Laponite Clay Suspensions. Philos. Mag. 2008, 88 (33-35), 4213-4221. https://doi.org/10.1080/14786430802581876.

(24) Pozzo, D. C.; Walker, L. M. Reversible Shear Gelation of Polymer-Clay Dispersions. Colloid Surf. A-Physicochem. Eng. Asp. 2004, 240 (1-3), 187-198. https://doi.org/10.1016/j.colsurfa.2004.04.040.

(25) Zebrowski, J.; Prasad, V.; Zhang, W.; Walker, L. M.; Weitz, D. A. Shake-Gels: ShearInduced Gelation of Laponite-PEO Mixtures. Colloid Surf. A-Physicochem. Eng. Asp. 2003, 213 (2-3), 189-197. https://doi.org/10.1016/S0927-7757(02)00512-5.

(26) Gabriel, J. C. P.; Sanchez, C.; Davidson, P. Observation of Nematic Liquid-Crystal Textures in Aqueous Gels of Smectite Clays. J. Phys. Chem. 1996, 100 (26), 1113911143. https://doi.org/10.1021/jp961088z.

(27) Michot, L. J.; Bihannic, I.; Maddi, S.; Funari, S. S.; Baravian, C.; Levitz, P.; Davidson, P. Liquid-Crystalline Aqueous Clay Suspensions. Proc. Natl. Acad. Sci. U. S. A. 2006, 103 (44), 16101-16104. https://doi.org/10.1073/pnas.0605201103.

(28) Paineau, E.; Antonova, K.; Baravian, C.; Bihannic, I.; Davidson, P.; Dozov, I.; ImperorClerc, M.; Levitz, P.; Madsen, A.; Meneau, F.; Michot, L. J. Liquid-Crystalline Nematic Phase in Aqueous Suspensions of a Disk-Shaped Natural Beidellite Clay. J. Phys. Chem. B 2009, 113 (48), 15858-15869. https://doi.org/10.1021/jp908326y.

(29) Paineau, E.; Philippe, A. M.; Antonova, K.; Bihannic, I.; Davidson, P.; Dozov, I.; Gabriel, J. C. P.; Imperor-Clerc, M.; Levitz, P.; Meneau, F.; Michot, L. J. Liquid-Crystalline 
Properties of Aqueous Suspensions of Natural Clay Nanosheets. Liq. Cryst. Rev. 2013, 1 (2), 110-126. https://doi.org/10.1080/21680396.2013.842130.

(30) Lemaire, B. J.; Panine, P.; Gabriel, J. C. P.; Davidson, P. The Measurement by SAXS of the Nematic Order Parameter of Laponite Gels. Europhys. Lett. 2002, 59 (1), 55-61. https://doi.org/10.1209/ep1/i2002-00159-8.

(31) Yuang, P. C.; Shen, Y. H. Determination of the Surface Area of Smectite in Water by Ethylene Oxide Chain Adsorption. J. Colloid Interface Sci. 2005, 285 (2), 443-447. https://doi.org/10.1016/j.jcis.2004.12.056.

(32) Rossi, S.; Luckham, P. F.; Tadros, T. F. Influence of Non-Ionic Polymers on the Rheological Behaviour of Na+-Montmorillonite Clay Suspensions. Part II. Homopolymer Ethyleneoxide and Polypropylene Oxidepolyethylene Oxide ABA Copolymers. Colloid Surf. A-Physicochem. Eng. Asp. 2003, 215 (1-3), 1-10. https://doi.org/10.1016/S09277757(02)00446-6.

(33) Feigin, L. A.; Svergun, D. I. Structure Analysis by Small-Angle X-Ray and Neutron Scattering; Taylor, G. W., Ed.; Springer US: Boston, MA, 1987. https://doi.org/10.1007/978-1-4757-6624-0.

(34) Burchill, S.; Hall, P.; Harrison, R.; Hayes, M.; Langford, J.; Livingston, W.; Smedley, R.; Ross, D.; Tuck, J. Smectite Polymer Interactions in Aqueous Systems. Clay Min. 1983, 18 (4), 373-397. https://doi.org/10.1180/claymin.1983.018.4.04.

(35) Lekkerkerker, H. N. W.; Tuinier, R. Colloids and the Depletion Interaction; Lecture Notes in Physics; Springer Netherlands, 2011. https://doi.org/10.1007/978-94-007-1223-2.

(36) Zhang, S. D.; Reynolds, P. A.; van Duijneveldt, J. S. Phase Behavior of Mixtures of Colloidal Platelets and Nonadsorbing Polymers. J. Chem. Phys. 2002, 117 (21), 99479958. https://doi.org/10.1063/1.1518007.

(37) Kuhl, T. L.; Leckband, D. E.; Lasic, D. D.; Israelachvili, J. N. Modulation of Interaction Forces between Bilayers Exposing Short-Chained Ethylene Oxide Headgroups. Biophysical Journal 1994, 66 (5), 1479-1488. https://doi.org/10.1016/S00063495(94)80938-5.

(38) Devanand, K.; Selser, J. C. Asymptotic Behavior and Long-Range Interactions in Aqueous Solutions of Poly(Ethylene Oxide). Macromolecules 1991, 24 (22), 5943-5947. https://doi.org/10.1021/ma00022a008.

(39) Fleer, G.; Stuart, M. A. C.; Scheutjens, J. M. H. M.; Cosgrove, T.; Vincent, B. Polymers at Interfaces; Springer Netherlands, 1998. https://doi.org/10.1007/978-94-011-2130-9.

(40) Liu, C.; Ewert, K. K.; Wonder, E.; Kohl, P.; Li, Y.; Qiao, W.; Safinya, C. R. Reversible Control of Spacing in Charged Lamellar Membrane Hydrogels by Hydrophobically Mediated Tethering with Symmetric and Asymmetric Double-End-Anchored Poly(Ethylene Glycol)s. ACS Appl. Mater. Interfaces 2018, 10 (50), 44152-44162. https://doi.org/10.1021/acsami.8b16456.

(41) Shundyak, K.; van Roij, R.; van der Schoot, P. Theory of the Isotropic-Nematic Transition in Dispersions of Compressible Rods. Phys. Rev. $E$ 2006, 74 (2), 021710. https://doi.org/10.1103/PhysRevE.74.021710.

(42) Duncan, P. D.; Dennison, M.; Masters, A. J.; Wilson, M. R. Theory and Computer Simulation for the Cubatic Phase of Cut Spheres. Phys. Rev. E 2009, 79 (3), 031702. https://doi.org/10.1103/PhysRevE.79.031702.

(43) Masters, A. J. Virial Expansions. J. Phys.: Condens. Matter 2008, 20 (28), 283102. https://doi.org/10.1088/0953-8984/20/28/283102. 
(44) Vroege, G.; Lekkerkerker, H. Phase-Transitions in Lyotropic Colloidal and Polymer Liquid-Crystals. Rep. Prog. Phys. 1992, 55 (8), 1241-1309. https://doi.org/10.1088/00344885/55/8/003.

(45) Onsager, L. The Effects of Shape on the Interaction of Colloidal Particles. Ann.NY Acad.Sci. 1949, 51 (4), 627-659. https://doi.org/10.1111/j.1749-6632.1949.tb27296.x.

(46) Wensink, H. H.; Vroege, G. J.; Lekkerkerker, H. N. W. Isotropic-Nematic Density Inversion in a Binary Mixture of Thin and Thick Hard Platelets. J. Phys. Chem. B 2001, 105 (43), 10610-10618. https://doi.org/10.1021/jp0105894.

(47) Jabbari-Farouji, S.; Weis, J.-J.; Davidson, P.; Levitz, P.; Trizac, E. Interplay of Anisotropy in Shape and Interactions in Charged Platelet Suspensions. J. Chem. Phys. 2014, 141 (22), 224510. https://doi.org/10.1063/1.4903045.

(48) Wensink, H. H.; Vroege, G. J. Phase Equilibria in Systems of Hard Disks with Thickness $\begin{array}{lllllll}\text { Polydispersity. Phys. Rev. } & \text { E 2002, } & 65 & \text { (3), } & 031716 .\end{array}$ https://doi.org/10.1103/PhysRevE.65.031716.

(49) Ruiz-Hitzky, E.; Aranda, P. Polymer-Salt Intercalation Complexes in Layer Silicates. Adv. Mater. 1990, 2 (11), 545-547. https://doi.org/10.1002/adma.19900021108.

(50) Montarges, E.; Michot, L.; Lhote, F.; Fabien, T.; Villieras, F. Intercalation of Al-13Polyethyleneoxide Complexes into Montmorillonite Clay. Clay Clay Min. 1995, 43 (4), 417-426. https://doi.org/10.1346/CCMN.1995.0430404.

(51) Aranda, P.; Ruiz-Hitzky, E. Poly(Ethylene Oxide)-Silicate Intercalation Materials. Chem. Mater. 1992, 4 (6), 1395-1403. https://doi.org/10.1021/cm00024a048.

(52) Gennes (de), P. G.; Gennes, P.-G. de. The Physics of Liquid Crystals; Clarendon Press, 1974. 
Table of Contents graphic:

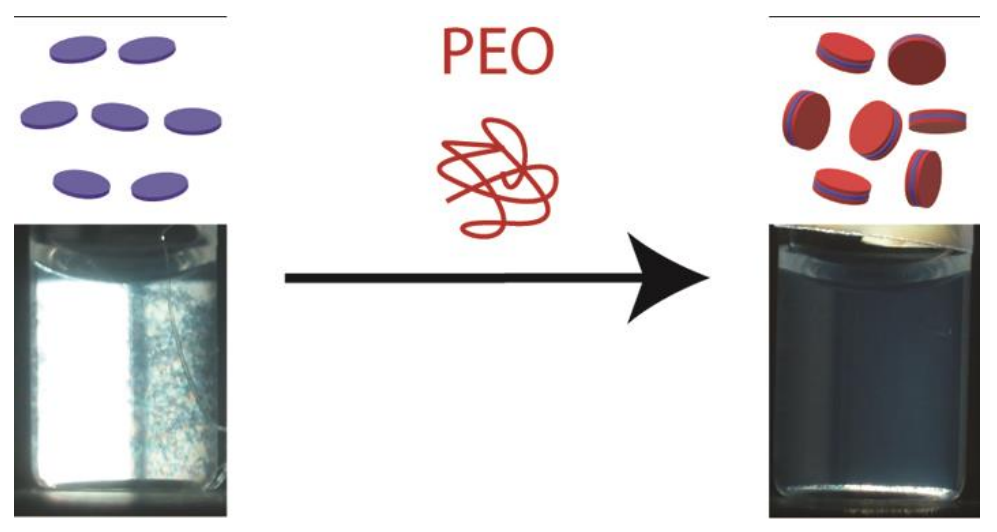

